[0212-7199 (2007) 24: 11; pp 554-557] ANALES DE MEDICINA INTERNA Copyright $(C) 2007$ ARAN EDICIONES, S.L.

AN. MED. INTERNA (Madrid) Vol. 24, N. ${ }^{\circ} 11$, pp. 554-557, 2007

\title{
Manejo del dolor oncológico
}

\author{
P. KHOSRAVI SHAHI, A. DEL CASTILLO RUEDA', G. PÉREZ MANGA \\ Servicio de Oncología Médica. ${ }^{I}$ Servicio de Medicina Interna II. Hospital General \\ Universitario Gregorio Marañón. Madrid
}

MANAGEMENT OF CANCER PAIN

\section{RESUMEN}

El dolor es un problema muy frecuente en los pacientes con cáncer, con una prevalencia del $90 \%$ en los estadios avanzados. El manejo del dolor oncológico es complejo, y un enfoque multidimensional es preciso para su manejo óptimo. Tres tipos de dolor ha sido descrito en base a la neurofisiología del dolor (Dolor somático, visceral y neuropático). Numerosas guías clínicas para el manejo del dolor oncológico han sido editadas por diversos investigadores y organizaciones. La guía clínica de la OMS es el más usado en el manejo del dolor oncológico. Los opioides, de los cuales la morfina es el prototipo, son los fármacos más importantes en el tratamiento del dolor oncológico. Los fármacos coadyuvantes son usados en el tratamiento del dolor oncológico, con el objetivo de potenciar la eficacia de los opioides, y para tratar algunos tipos específicos del dolor (dolor neuropático y óseo).

PALABRAS CLAVE: Dolor. Cáncer. Opioides. EVA. Neuropático.

\begin{abstract}
Pain is a very frequent problem in patients with cancer, with a prevalence of $90 \%$ in advanced disease. The management of cancer pain is complex, and a multidimensional approach is necessary for its optimal treatment. Three types of pain have been described based on the neurophysiology of pain pathways (somatic, visceral and neuropathic pain). Numerous guidelines for the management of cancer pain have been issued by various organizations and researchers. The WHO guideline is the most used in the management of pain cancer.The opioids analgesics, of which morphine is the prototype, are the most important drugs in the treatment of cancer pain. Adjuvant drugs are used in the treatment of cancer pain, in order to enhace opioid analgesia, and provide analgesia for certain specific types of pain (neuropathic pain, and bone pain).
\end{abstract}

KEY WORDS: Pain. Cancer. Opioids. VAS. Neuropathic.

Khosravi Shahi P, del Castillo Rueda A, Pérez Manga G. Manejo del dolor oncológico. An Med Interna (Madrid) 2007; $24: 554-557$.

\section{INTRODUCCIÓN}

La definición del dolor es compleja. La International Association of the Study of Pain lo define como una experiencia sensorial y emocional desagradable asociada o no a una lesión tisular, o que se describe con las manifestaciones propias de tal lesión. Esta definición confiere una visión multidimensional al dolor. De esta manera, el dolor no es exclusivamente una sensación debida a la estimulación de los nociceptores, sino que también implica la existencia de un factor emocional.

El dolor es un síntoma muy frecuente en el paciente oncológico, apareciendo hasta en el $90 \%$ de los pacientes con un cáncer terminal (1-3). El dolor oncológico es un problema complejo y de gran importancia en la práctica clínica diaria, que requiere un tratamiento multidimensional. La mayoría de los autores están de acuerdo con el empleo de la distintas guías clínicas existentes para el manejo del dolor oncológico como la de la Organización Mundial de la Salud (OMS) (4), la del Agency for Health Care Policy and Research (AHCPR) (5), o la del National Comprehensive Cancer Network (NCCN) (6). De esta forma, el dolor oncológico puede ser aliviado aproximadamente en el $90 \%$ de los casos mediante el uso correcto, y a menudo combinado de los tratamientos farmacológicos y no farmacológicos.

\section{CAUSAS DEL DOLOR ONCOLÓGICO}

Las causas del dolor oncológico son muy variadas, y habitualmente en el paciente con cáncer hay múltiples causas y localizaciones de dolor (7). 
Las principales causas del dolor oncológico son:

- La invasión tumoral de estructuras adyacentes (70\%): Como puede ser la invasión de estructuras óseas, invasión de elementos vasculares o nerviosos, obstrucción intestinal o la infiltración de vísceras huecas.

- Procedimientos diagnósticos y terapéuticos (20\%): Entre los que cabe mencionar las intervenciones quirúrgicas, los efectos secundarios propios de la quimioterapia (mucositis gastrointestinal y bucofaríngea, neurotoxicidad) y/o de la radioterapia (enteritis rádica, cistitis rádica, osteonecrosis rádica).

- Síndromes inducidos por la neoplasia $(<10 \%)$ : Como son los síndromes paraneoplásicos.

-Otras causas extraoncológicas: como la artrosis, osteoporosis, cardiopatía isquémica, infarto de miocardio, etc...

\section{CLASIFICACIÓN DEL DOLOR ONCOLÓGICO}

El dolor oncológico puede ser clasificado de múltiples formas, teniendo en cuenta el mecanismo de producción, su duración en el tiempo, o su localización (Tabla I).

\section{TABLA I}

\section{CLASIFICACIÓN DEL DOLOR ONCOLÓGICO}

\begin{tabular}{l} 
Mecanismo de producción \\
Dolor nociceptivo \\
Dolor neurógeno \\
Dolor psicógeno \\
Localización \\
Dolor fantasma \\
Dolor somático \\
Dolor visceral \\
Duración en el tiempo \\
Dolor agudo \\
Dolor crónico \\
\hline
\end{tabular}

Clásicamente el dolor se ha clasificado en tres grandes subtipos, en base a la neurofisiología y neuroanatomía del dolor (8):

-Dolor somático: Es el dolor que se produce como consecuencia de la activación de los nociceptores en la piel o en los tejidos profundos. Suele tratarse de un dolor bien localizado. Algunos ejemplos de este tipo de dolor son el dolor por metástasis óseas (9), el dolor músculo-esquelético y miofascial.

-Dolor visceral: El dolor visceral surge tras la activación de los nociceptores por infiltración y/o compresión de vísceras torácicas, abdominales o pélvicas. Típicamente aparece en la carcinomatosis peritoneal, sobre todo en el seno del cáncer pancreático. Se trata de un dolor difuso y mal localizado. En su fisiopatogenia parece jugar un papel importante los receptores opioides kappa (10).

-Dolor neuropático: El dolor neuropático está causado por una lesión primaria o por una disfunción en el sistema nervioso central o periférico, con destrucción y/o afectación nerviosa. Este tipo de dolor generalmente se describe como descargas paroxísticas con sensación de ardor o quemazón, o bien como pinchazos o entumecimiento y hormigueo.

Otra forma de clasificar el dolor oncológico es en función de la duración del mismo en el tiempo:
-Dolor agudo: Es un dolor de corta duración (duración inferior a 6 meses), y generalmente de aparición brusca.

-Dolor crónico: Es el dolor que suele aparecer de forma lenta, y habitualmente tiene una duración en el tiempo superior a los 6 meses.

\section{EVALUACIÓN DEL DOLOR ONCOLÓGICO}

Previo al inicio del tratamiento del dolor en los pacientes oncológicos es preciso realizar una valoración multidimensional del dolor, en un intento de indagar sobre los mecanismos fisiopatológicos, y otros factores asociados al dolor como los emocionales y psicológicos (11).

Es preciso valorar de forma exhaustiva una serie de aspectos relacionados con la percepción del dolor por parte del paciente oncológico:

-Intensidad del dolor: La intensidad del dolor tiene un carácter muy subjetivo, y para su valoración existen distintas escalas de evaluación.

- Tipo de dolor: Es conveniente diferenciar entre el dolor somático, visceral, neuropático o mixto, puesto que cada tipo de dolor puede requerir un tratamiento distinto. Ya que por ejemplo, el dolor neuropático es mucho más resistente a los opioides clásicos como la codeína, morfina o el fentanilo, y aparentemente responde mejor a la oxicodona, buprenorfina y sobre todo a la metadona (12). Además los fármacos coadyuvantes como la gabapentina, venlafaxina, amitriptilina, carbamacepina o pregabalina son útiles en el tratamiento del dolor neuropático.

-Patrón del dolor: Hace referencia al momento del comienzo del dolor, su duración o persistencia.

- Factores desencadenantes: Es necesario identificar los posibles factores que precipitan el cuadro del dolor para evitarlos, como pueden ser la humedad, el frío, el movimiento, la ansiedad, la tensión física o emocional.

- Factores de alivio: Existen distintas circunstancias que puede mitigar el dolor oncológico como el descanso, la aplicación de calor o frío y la medicación analgésica.

- Síntomas que acompañan al dolor oncológico: Distintos síntomas puede asociarse al cuadro del dolor como las náuseas, los vómitos, las diarreas o el mareo.

- Efectos del dolor sobre las actividades de la vida diaria: El dolor oncológico puede conllevar con frecuencia una limitación de las actividades de la vida diaria, como la imposibilidad para el aseo personal, afectación de las relaciones sociales y familiares, o la dificultad para la deambulación, que puede llevar a una mayor depresión del estado del ánimo, y ésta a su vez causar un mayor dolor.

-Examen físico del paciente oncológico: Con el examen físico podemos analizar las respuestas fisiológicas del paciente ante el dolor y las respuestas conductuales.

Finalmente, es preciso mencionar que para un correcto control del dolor oncológico hay que tener en cuenta una serie de acciones básicas:

- Apoyo psicosocial óptimo al paciente oncológico.

-Uso adecuado de los fármacos analgésicos y de los coadyuvantes, teniendo presente la escalera analgésica del dolor de la OMS (Fig. 1) (13).

-Explicación de las causas del dolor al paciente y a la familia, para que de esta forma puedan atajarlo de la manera más eficaz posible. 


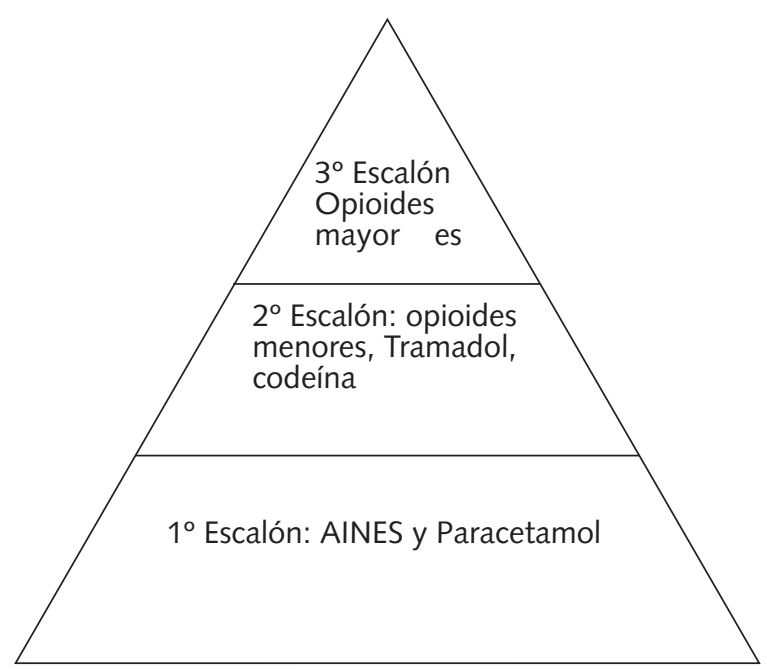

Fig. 1. Escalera Analgésica de la OMS.

-Uso de los tratamientos oncológicos activos específicos para cada proceso neoplásico.

- Reservar los procedimientos invasivos dirigidos al bloqueo de la transmisión central del dolor para aquellos pacientes que no hayan respondido a las medidas más conservadoras.

\section{ESCALAS DE VALORACIÓN DEL DOLOR}

Las escalas de valoración del dolor son métodos clásicos de medición de la intensidad del dolor, y con su empleo podemos llegar a cuantificar la percepción subjetiva del dolor por parte del paciente. Con ello, podemos ajustar de una forma más exacta el uso de los fármacos analgésicos. Puesto que, recientemente se recomienda obviar el paso escalonado de los analgésicos, establecido por la OMS, en los casos en los que los pacientes refieran un dolor severo de inicio. Así se debería de iniciar directamente con el tercer escalón de la analgesia sin necesidad de ensayar los dos escalones previos con el objetivo de evitar períodos de dolor mal controlado.

Estas escalas de valoración del dolor oncológico son generalmente de fácil manejo y entendimiento por parte de los pacientes, con lo que se recomienda su utilización en la práctica clínica diaria. Existen diferentes escalas del valoración del dolor:

- Escalas verbales o de descriptores verbales: Mediante el uso de estas escalas se evalúan la intensidad del dolor y el grado de mejoría que experimenta el paciente oncológico, a partir de palabras que expresan diversos grados de intensidad, bien sea en orden decreciente o bien creciente. El número de niveles puede variar de 4 a 15 .

-Escala gráfica: Se trata de una escala analógica visual en la que se indican números o adjetivos a lo largo de una línea, definiendo diversos niveles de intensidad del dolor. Este tipo de escala también puede utilizarse para comprobar el grado de efectividad del tratamiento.

- Escala analógica visual (EVA): Esta escala de dolor fue ideada por Aitken en 1969, y consiste en una línea horizontal o vertical de 10 centímetros de longitud, cuyos extremos están señaladas con los términos "no dolor" y "dolor insoportable". Y es el paciente el que colocará la cruz en la línea en función del dolor que experimente. La EVA también puede ser usado para valorar la respuesta al tratamiento analgésico (Fig. 2).

- Escalas visuales para los niños: Este tipo de escala de evaluación del dolor fue creado por Won en 1986. Este tipo de escalas está formado habitualmente por 6 expresiones faciales, siendo la primera cara muy sonriente y la última triste y llorosa, mientras que los rostros intermedios muestran diferentes grados de felicidad y tristeza (Fig. 3).

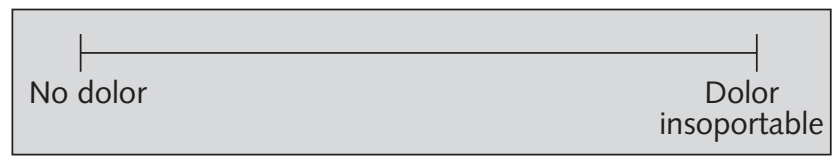

Fig. 2. Escalera Analógica Visual (EVA).

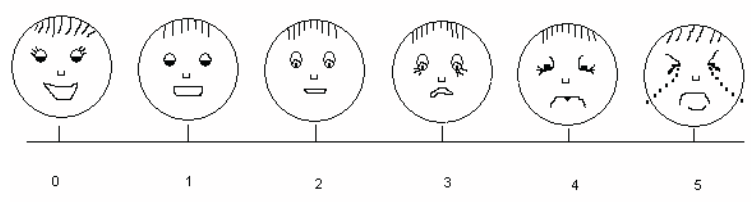

Fig. 3. Escalas de caras de Wong para los niños.

\section{TRATAMIENTO DEL DOLOR ONCOLÓGICO}

El tratamiento del dolor oncológico debe incidir en los distintos aspectos del dolor, incluyendo las posibles causas, factores desencadenantes o de alivio, el estado de ánimo y la dimensión pisco-social del paciente, el tipo de dolor, y la intensidad del dolor.

El tratamiento analgésico del dolor oncológico dependerá sobre todo del tipo de dolor y de la intensidad. De manera que en los dolores con EVA igual o superior a siete se debería de iniciar de entrada con el tercer escalón de la analgesia, obviando los dos escalones previos. La vía de administración recomendada es la vía oral, y en los pacientes con dificultades para la deglución se puede recurrir a las formas transdérmicas (TD), y en determinadas circunstancias la vía parenteral es la preferible (14).

El primer escalón de la analgesia desarrollada por la OMS está formado por paracetamol y los antiinflamatorios no esteroideos (AINES), y su uso está indicado solamente en casos de dolor oncológico leve o moderado. Este primer escalón al igual que los otros dos se puede asociar a los fármacos coadyuvantes. Además el primer escalón se puede combinar con el segundo o tercer escalón. El paracetamol en los pacientes oncológicos debe de usarse a una dosis de $1 \mathrm{gr} / 8$ horas por vía oral (VO). El grupo de AINES está formado por un amplio número de fármacos, entre los que cabe destacar el ibuprofeno a dosis de $600 \mathrm{mg} / 8$ horas por VO, sobre todo en el tratamiento de las metástasis óseas. No se recomienda la combinación de dos o más AINES entre sí, pero es posible asociar el paracetamol con un AINE.

El segundo escalón está formado por los opioides menores, entre los que cabe destacar la codeína y el tramadol. Sin embargo, cabe mencionar que el efecto analgésico de la codeína es débil, y no se recomienda su uso como tal, ya que además de su escasa eficacia analgésica es muy frecuente el estre- 
ñimiento que deriva de su uso. El fármaco de elección en este escalón es el tramadol que puede usarse a dosis de 75-400 $\mathrm{mg}$ /día repartidas en 3 ó 4 tomas por VO. La buprenorfina por vía TD a pesar de ser clasificado como un opioide mayor, debería de incluirse en este segundo escalón por su baja eficacia analgésica en los dolores oncológicos intensos.

El tercer escalón analgésico es la "estrella" en el manejo habitual del dolor oncológico (15), y su empleo está indicado tras el fracaso escalonado de la analgesia o bien de inicio ante la presencia de dolores muy severos $(\mathrm{EVA}>6)$. Como norma general nunca debe de asociarse fármacos de segundo y tercer escalón, ya que su combinación no aumenta la eficacia analgésica, y sin embargo supone un aumento de la toxicidad. El opioide mayor de referencia es la morfina, del que existen formas de liberación rápida y sostenida (sulfato de morfina) por VO e incluso a través de la sonda nasogástrica. Otros opioides mayores empleados con frecuencia en nuestro medio son el fentanilo por vía TD (liberación sostenida) o transmucosa (liberación rápida) (16), metadona por VO, buprenorfina TD, y más recientemente la oxicodona por VO. Los fármacos del tercer escalón no tienen un techo analgésico, y la dosis limitante viene determinado por la aparición de efectos secundarios graves como la depresión respiratoria, la sedación excesiva o el síndrome de neurotoxicidad inducida por opioides (SNIO) (17).

Además de los fármacos analgésicos mencionados se debe de tener en cuenta el uso concomitante de los fármacos adyuvantes, que en ocasiones resultan esenciales en el manejo del dolor oncológico, como ocurre en el caso del dolor neuropático (18) o de las metástasis óseas. Son muchos los fármacos coadyuvantes que se pueden emplear en el tratamiento del dolor oncológico (19-21) (Tabla II).

Por último, cabe mencionar la existencia de otros muchos procedimientos para lograr el alivio del dolor oncológico como puede ser el uso de los bifosfonatos endovenosos (zolendrona- to) (22) en el tratamiento de las metástasis óseas por cáncer de mama o de próstata (23); situaciones en los que también resultan de utilidad el uso de radiofármacos como Samario-153 y Estroncio-90 (24). La radioterapia paliativa antiálgica resulta de mucha efectividad sobre las metástasis óseas o las masas de partes blandas que provocan compresión medular y/o radicular. Otros métodos efectivos son las técnicas neuroablativas usado en los casos más resistentes a los fármacos analgésicos, el apoyo psicológico y la psicoterapia, la acupuntura, las técnicas de relajación, y las cirugías con intención antiálgica.

\section{TABLA I}

FÁRMACOS COADYUVANTES EN EL DOLOR ONCOLÓGICO

\begin{tabular}{ll}
\hline Fármaco & Indicación \\
\hline Antidepresivos & \\
Amitriptilina & Dolor neuropático \\
Paroxetina & Depresión asociada a ansiedad \\
Fluoxetina & Depresión asociada a inhibición \\
Venlafaxina & Depresión y dolor neuropático \\
Anticonvulsivantes & \\
Carmabacepina & Dolor neuropático \\
Cabapentina & Dolor neuropático \\
Corticoides & Metástasis óseas, compresión medular, hepatalgia, \\
& hipertensión intracraneal \\
Benzodiacepinas & Tratamiento de la ansiedad asociada al dolor \\
Anestésicos & \\
Ketamina & Dolor neuropático \\
Lidocaína viscosa 1\% & Dolor por mucositis bucofaríngea postquimiotera- \\
& pia/radioterapia \\
\hline
\end{tabular}

\section{Bibliografía}

1. Foley KM. Pain assessment and cancer pain syndromes. In: Doyle D, Hanks GWC, Calman K, Cherny N, eds. Oxford textbook of palliative medicine, $3^{\text {rd }}$ ed. New York: Oxford University Press 2003.

2. Levy MH. Pharmacologic treatment of cancer pain. N Engl J Med 1996; 335: 1124-32.

3. Sepulveda C, Marlin A, Yoshida T, Ulrich A. Palliative care: the World Health Organization's global perspective. J Pain Symptom Manage 2002; 24: 91-6

4. WHO Expert Committee.Cancer pain relief. World Health Organization. Geneva 1986.

5. Jacox AK, Carr DB, Payne R, et al. Management of cancer pain. Clinical practice guideline no. 9. AHCPR publication no. 94-0592, Agency for Health Care Policy and Research, Rockville, MD 1994.

6. Benedetti C, Brock C, Cleeland C, et al. National Comprehensive Cancer Network. NCCN practice guidelines for cancer pain. Oncol 2000; 14: $135-50$.

7. Bruera E, Kim HN. Cancer pain. JAMA 2003; 290: 2476-9.

8. Besson JM. The neurobiology of pain. Lancet 1999; 353: 1610-15.

9. Clohisy DR, Mantyh PW. Bone cancer pain. Cancer 2003; 97: 866-73.

10. Cervero F, Laird JMA. Pain: Visceral pain. Lancet 1999; 353: 2145-8.

11. Stjernsward J. Palliative medicine: A global perspective. In: Doyle D, Hanks G, Cherny N, eds. Oxford textbook of palliative medicine, 3rd ed. New York: Oxford University Press 2003.

12. Foley KM. Opioids and chronic neuropathic pain. N Engl J Med 2003; 348: $1279-81$

13. Zech D, Grond S, Lynch J, Hertel D, Lehmann K. Validation of World Health Organization guidelines for cancer pain relief: A 10 year prospective study, Pain 1995; 63: 65-76.

14. Aliaga L, Baños JE, Barutell C, Molet J. Tratamiento del dolor. Teoría y práctica. Editorial Permanyer 2002.

15. McQuay H. Opioids in pain management. Lancet 1999; 353: 2229-32.

16. Portenoy RK, Payne R, Coluzzi P, et al. Oral transmucosal fentanyl citrate (OTFC) for the treatment of breakthrough pain in cancer patients, a controlled dose titration study. Pain 1999; 79: 303-12.

17. Aliaga L, Catala E. Opioides. Utilización Clínica. You \& US, 2001.

18. Stute P, Soukup J, Menzel M, Sabatowski R, Grond S. Analysis and treatment of different types of neuropathic cancer pain. J Pain Symptom Manage 2003; 26: 1123-31.

19. Portenoy RK. Adjuvant analgesic agents. Hematol Oncol Clin North Am 1996; 10: 103-19.

20. Backonja M, Beydoun A, Edwards KR, et al. Gabapentin for the symptomatic treatment of painful neuropathy in diabetes mellitus, a randomized controlled trial. JAMA 1998; 280: 1831-6.

21. Slatkin NS, Rhiner M. Ketamine in the treatment of refractory cancer pain. J Support Oncol 2003; 14: 287-93.

22. Hernández JL, Riancho JA, González J. Bifosfonatos intravenosos. Med Clin (Barc) 2005; 124: 384-54.

23. Khosravi P, Díaz VM. Bifosfonatos en oncología. An Med Interna (Madrid) 2005; 22: 544-547.

24. Khosravi P, Pérez Manga G. Cáncer de próstata metastático. Oncología 2006; 29: 16-22. 